\title{
Low correlation between visit-to-visit variability and 24-h variability of blood pressure
}

\author{
Paul Muntner', Daichi Shimbo², Keith M Diaz², Jonathan Newman², Richard P Sloan ${ }^{2}$ \\ and Joseph E Schwartz ${ }^{2,3}$
}

visit-to-visit variability (VVV) of clinic systolic blood pressure (SBP) has been associated with cardiovascular disease risk. Given the need for obtaining blood pressure (BP) at multiple visits to calculate VVV, substituting BP variability from ambulatory blood pressure monitoring (ABPM) may be a practical alternative. We assessed the correlation between VVV of BP and BP variability from ABPM using data from 146 untreated, mostly normotensive participants (mean age 47.9 years), in a substudy of the ongoing Masked Hypertension Study. VVV of SBP and diastolic blood pressure (DBP) was estimated by the standard deviation (s.d $\left.\mathrm{v}_{\mathrm{vvv}}\right)$ and average real variability $\left(\mathrm{ARV}_{\mathrm{vvv}}\right)$ from six study visits over a median of 216 days. ABPM data were used to calculate the day-night s.d. (s.d.dn), and the ARV of SBP and DBP over $24 \mathrm{~h}\left(\mathrm{ARV}_{24}\right)$. For SBP, the mean

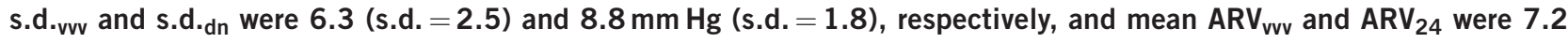
(s.d. $=3.2$ ) and $8.4 \mathrm{~mm} \mathrm{Hg}$ (s.d. $=2.1$ ), respectively. Spearman's correlation coefficient between s.d.vvv and s.d.dn of SBP was $r_{\mathrm{s}}=0.25$, and between $\mathrm{ARV}_{\mathrm{vvv}}$ and $\mathrm{ARV}_{24}$ was $r_{\mathrm{s}}=0.17$. Participants in the highest quartile of $\mathrm{s} . \mathrm{d}_{\mathrm{dn}}$ of SBP were 1.66 (95\% Cl: 0.93-2.75) times more likely to be in the highest quartile of s.d.vvv of SBP. The observed-to-expected ratio between the highest quartiles of $A_{R V}$ vvv $A_{\mathrm{vVV}}$ of $\mathrm{SBP}$ was $0.89(95 \% \mathrm{Cl}: 0.41-1.69)$. The correlations for s.d.vvv and s.d.dn, and $A R V_{v v v}$ and $A R V_{24}$ of DBP were minimal. These data suggest VVV and 24-h variability are weakly correlated and not interchangeable.

Hypertension Research (2013) 36, 940-946; doi:10.1038/hr.2013.58; published online 20 June 2013

Keywords: blood pressure variability; reliability; ambulatory blood pressure monitoring; blood pressure measurement; methods

Ambulatory blood pressure monitoring (ABPM) is considered to provide a more accurate estimate of a patient's mean systolic and diastolic blood pressure (SBP and DBP) compared with clinic measurements. ${ }^{1,2}$ Also, mean blood pressure (BP) from ABPM is a stronger risk factor for cardiovascular disease (CVD) compared with mean clinic $\mathrm{BP} .^{3-5}$ The value of ABPM for the measurement of BP is highlighted in the 2011 NICE guidelines, which recommend using it to confirm the diagnosis of hypertension for patients with an SBP or $\mathrm{DBP} \geqslant 140$ or $90 \mathrm{~mm} \mathrm{Hg}$, respectively. 6,7

Several recent studies have reported strong associations between visit-to-visit variability (VVV) of clinic SBP and the occurrence of stroke, coronary heart disease and all-cause mortality. 8,9 This has created substantial interest in BP variability as a novel risk factor and as a possible target for interventions to reduce CVD risk. ${ }^{10,11}$ However, calculating the VVV of BP requires multiple visits, and therefore is not currently done in clinical practice. Substituting BP variability from ABPM for VVV of BP may be a practical alternative. However, a meta-analysis of 8938 individuals demonstrated weak or no associations between measures of BP variability from ABPM (for example, day-night s.d., s.d.dn, and average real variability over $\left.24 \mathrm{~h}, \mathrm{ARV}_{24}\right), \mathrm{CVD}$ incidence and all-cause mortality. ${ }^{12}$ Although these data suggest that the VVV of BP and ABPM measures of BP variability represent different underlying constructs, few data are available on the relationship of BP variability derived from ABPM to that derived across multiple clinic visits.

The goal of the current study was to assess the association between VVV and ABPM measures of BP variability. Additionally, we determined correlates of VVV and ABPM variability of BP. To accomplish these goals, we analyzed data on SBP and DBP from a substudy of the Masked Hypertension Study.

\section{METHODS}

The Masked Hypertension Study, an ongoing study of the prevalence, predictors and prognosis of masked hypertension, is comprised of employees recruited from Stony Brook University, Stony Brook University Hospital, Columbia University Medical Center and a private hedge fund management organization in New York. The study was restricted to individuals $\geqslant 18$ years of age who were not taking antihypertensive or other medications that are known to affect blood pressure. Additionally, participants were deemed ineligible if they had a history of CVD or major arrhythmias, evidence of secondary hypertension other than a history of pregnancy-induced hypertension, a serum

${ }^{1}$ Department of Epidemology, University of Alabama at Birmingham, Birmingham, AL, USA; ${ }^{2}$ Department of Medicine, Columbia University, New York, NY, USA and ${ }^{3}$ Department of Psychiatry and Behavioral Sciences, Stony Brook University, Stony Brook, NY, USA

Correspondence: Dr P Muntner, Department of Epidemiology, University of Alabama at Birmingham, 1665 University Boulevard, Suite 230J, Birmingham, AL 35294, USA E-mail: pmuntner@uab.edu

Received 21 November 2012; revised 30 January 2013; accepted 28 March 2013; published online 20 June 2013 
creatinine $>1.6 \mathrm{mg} \mathrm{dl}^{-1}$, liver disease, adrenal disease or thyroid disease. Based on the average of their second and third BP measurements during an initial screening visit prior to enrollment, participants were required to have a clinic SBP $<160 \mathrm{~mm} \mathrm{Hg}$ and DBP $<105 \mathrm{~mm} \mathrm{Hg}$.

Of relevance to the current analysis, eligible participants attended five visits over a 4-week period. During each of the first three visits (visit 1-3), which were scheduled to occur over a 3-week period, clinic BP was measured. At the conclusion of visit 3, the participant was fitted with an ABPM device for BP monitoring over the subsequent $24 \mathrm{~h}$. At visit 4 , the next day, participants returned the ABPM device, and were given a plastic container and instructions for the collection of an overnight urine sample. At visit 5, CVD risk factor measures including anthropometrics were obtained. As part of a validation substudy, a randomly selected subset of 20\% of Masked Hypertension Study participants recruited at Stony Brook University or Stony Brook University Hospital completed a second set of four visits (visits 6-9) an average of 7 months (median of 6 months) after their visit 5 . For these participants, during visits $6-8$, which again were scheduled to occur over a 3 -week period, clinic BP was once again measured. The median duration between visits with clinic BP measurements was $7,11,165,7$ and 9 days for visits 1 to 2,2 to 3,3 to 6,6 to 7 and 7 to 8 , respectively. Overall, the median duration between visits 1 and 8 was 216 days.

Overall, 886 participants were enrolled between February 2005 and August 2011. For the current analysis, we included 174 (19.6\%) participants who completed the validation substudy visits, and thus could have six clinic visits with BP measurements. Of these participants, 16 did not have BP measurements at all six study visits, 10 were missing nighttime s.d. from ABPM, and 2 participants had $<80 \%$ valid ABPM measurements and were excluded from all analyses. After excluding these participants, there were 146 participants available for the current analysis. The Masked Hypertension Study and validation substudy were approved by the Institutional Review Boards at the participating institutions, and all participants provided written informed consent.

Of relevance to the current study, information on demographics (age, race, ethnicity and gender) was collected by standardized questionnaire. At visit 5, waist circumference was measured mid-way between the lowest rib and the iliac crest, with the participant standing. Also at visit 5, fasting blood samples were drawn from participants, and glycosylated hemoglobin (HbAlc) was measured using high-pressure liquid chromatography. Diabetes was defined as a fasting $\mathrm{HbAlc} \geqslant 6.5 \%$, current use of insulin or oral hypoglycemics, or a patient-reported diagnosis of diabetes by a physician. ${ }^{13}$ Among participants not using insulin or oral hypoglycemics and without a history of diabetes, impaired fasting glucose was defined as an HbAlc of $5.7-6.4 \%$, and normal glucose was defined as an $\mathrm{HbAlc}<5.7 \%$. Highsensitivity C-reactive protein (hsCRP) was measured by nephelometry, and levels $>3 \mathrm{mgl}^{-1}$ were defined as elevated. ${ }^{13}$ Using the urine collected the night before visit 5, urinary albumin and urinary creatinine were measured by nephelometry, and albuminuria was calculated as the albumin-tocreatinine ratio (ACR).

\section{Blood pressure measurements}

During visits $1-3$ and visits $6-8$, clinic BP was measured in a standardized manner consistent with published recommendations. ${ }^{14}$ Prior to clinic BP measurements being obtained, participants were asked to sit at rest for $5 \mathrm{~min}$ or longer. The arm circumference was measured, and appropriate-sized cuffs were utilized for BP assessment. A trained research nurse/technician obtained three sitting clinic BP readings, at 1-2 min intervals, using a Baum mercury sphygmomanometer (W.A. Baum, Copiague, NY, USA) and stethoscope. For each visit, the three measurements were averaged, and the six visit averages were used for all subsequent calculations and analyses.

At the conclusion of visit 3, participants were fit with an appropriate-sized arm cuff for the Spacelabs ABPM (Model 90207; Spacelabs, Redmond, WA, USA). ABP readings were taken at 28-min intervals throughout the following $24 \mathrm{~h}$. Recordings were analyzed to obtain average awake, and sleep SBP and DBP levels, based on times defined by data obtained from an actigraphy monitor worn on the wrist (ActiWatch; Phillips Respironics, Murrayville, PA,
USA), and supplemented by diary reports of the times participants woke up and went to sleep.

\section{Derivation of BP variability measures}

Two measures of VVV and ABPM variability were calculated for the current study. For VVV, we calculated the intra-individual s.d. and the ARV across the six visits. ARV takes into account the order in which the BP measurements were obtained, and is a measure of differences between adjacent visits. ${ }^{15}$ For ABPM, we calculated the s.d.dn and $\mathrm{ARV}_{24}$. The s.d. for daytime measurements and separately for nighttime measurements were calculated based on when participants were awake and sleeping, and the s.d.dn was calculated as the weighted mean of these s.d.'s. ${ }^{16}$ Weights were calculated as the duration of time that the participants were awake and sleeping. This approach is considered advantageous compared with calculating a single s.d. over $24 \mathrm{~h}$, as it eliminates the influence of the day-night change in BP. $\mathrm{ARV}_{24}$ was calculated as the average absolute difference between consecutive readings. ${ }^{17}$ The $\mathrm{ARV}_{24}$ accounts for the order of BP measurements over the ABP monitoring period. Day-night changes in SBP and DBP (that is, dipping) were calculated as the ratio of mean sleep-to-awake SBP and DBP, respectively.

\section{Statistical methods}

Participant characteristics were calculated as mean (95\% confidence interval) or percentage as appropriate. Given its non-Gaussian distribution, albuminuria is presented as geometric mean ( $95 \%$ confidence interval). Paired $t$-tests were used to compare the mean levels of clinic and ambulatory measures of mean BP and BP variability. Below we describe the analyses conducted for s.d. .vvv $_{\text {and }}$ s.d.dn of SBP. Identical analyses were conducted for s.d.vvv and s.d.dn of DBP, and for $\mathrm{ARV}_{\mathrm{vvv}}$ and $\mathrm{ARV}_{24}$ of SBP and DBP. Scatterplots between s.d. .vvv $_{\text {and }}$ s.d.dn of SBP were created, and Spearman's correlation coefficients were calculated. s.d. ${ }_{\mathrm{vvv}}$ and s.d.dn of SBP were divided into quartiles based on the distribution in the study sample. Weighted kappa statistics were used to calculate the agreement between quartiles. ${ }^{18}$ Among individuals in the highest quartile of s.d.dn, we calculated the observed and expected number of

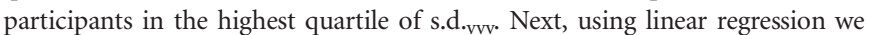
identified factors associated with s.d..$_{\mathrm{vvv}}$ and, separately, s.d.dn. Factors investigated included age, gender, race, ethnicity, diabetes status, abdominal obesity, elevated hsCRP, albuminuria and mean SBP across study visits for s.d. ${ }_{\mathrm{vvv}}$ and over $24 \mathrm{~h}$ for s.d.dn. Two sensitivity analyses were conducted. First, the correlation between daytime s.d. (s.d.day) with s.d. .vv $_{\text {of SBP and DBP was }}$ assessed. Additionally, the agreement between s.d.day, and s.d.vvv of SBP and DBP was calculated. Second, the correlation between sleep-to-awake ratio of SBP and DBP with s.d.vvv was determined. Additionally, the mean s.d.vvv was calculated for extreme dippers, dippers and non-dippers (sleep-to-awake ratio of SBP and DBP $<0.8,0.8$ to $\leqslant 0.9$ and $>0.9$, respectively). ${ }^{19} P$-values $<0.05$ were considered statistically significant. Analyses were conducted using SAS 9.2 (SAS Institute, Cary, NC, USA).

\section{RESULTS}

The mean age of the 146 study participants included in the current analyses was 47.9 years, $35.6 \%$ were men, $6.2 \%$ were black and $6.2 \%$ were hispanic (Table 1). The mean SBP from clinic and 24-h ABPM measurements was 115.2 (s.d. =10.9) and $117.6 \mathrm{~mm} \mathrm{Hg}($ s.d. =9.5), respectively $(P<0.001)$. The mean DBP was $74.7 \mathrm{~mm} \mathrm{Hg}($ s.d. $=7.3)$ for clinic measurements and $72.3 \mathrm{~mm} \mathrm{Hg}($ s.d. $=7.0)$ for the 24 -h ABPM measurements, respectively $(P<0.001)$.

visit-to-visit and day-night standard deviation of blood pressure For both SBP and DBP, the s.d. .vvv $_{\text {was lower than the s.d.dn }}$ (s.d. .vv $_{\text {vin }}$ and s.d.dn SBP: 6.3 and $8.8 \mathrm{~mm} \mathrm{Hg}$, respectively, and s.d. vvv $_{\text {din }}$ and s.d.dn DBP: 4.6 and $7.4 \mathrm{~mm} \mathrm{Hg}$, respectively; each $P<0.001$ ). The Spearman's correlation of s.d. ${ }_{\mathrm{vvv}}$ and s.d. dn $_{\text {n }}$ was 0.25 for SBP, and 0.02 for DBP (Figure 1). The weighted kappa for the concordance of quartiles of s.d. ${ }_{\mathrm{vvv}}$ and s.d.dn of SBP was 0.17 (Table 2, top panel). The analogous weighted kappa statistic for DBP was 0.01 (Table 2, bottom 
panel). Participants in the highest quartile of s.d.dn of SBP were more likely to be in the highest quartile of s.d. .vvv $_{\text {of }}$ SBP (observed-toexpected ratio $=1.66,95 \%$ CI: 0.93-2.75). For DBP, the observed-toexpected ratio for being in the highest quartile of s.d.dn and s.d.vvv was 1.11 (95\% CI: 0.56-1.98).

After multivariable adjustment for age, gender, race, ethnicity and mean clinic SBP, higher levels of albuminuria were associated with a

Table 1 Characteristics of participants in the Masked Hypertension Study

\begin{tabular}{|c|c|}
\hline Characteristics & Mean (s.d.) or percentage $(n=146)$ \\
\hline Age, years & $47.9(9.2)$ \\
\hline Men & $35.6 \%$ \\
\hline Black & $6.2 \%$ \\
\hline Hispanic & $6.2 \%$ \\
\hline \multicolumn{2}{|l|}{ Diabetes status } \\
\hline Normal fasting glucose & $58.6 \%$ \\
\hline Impaired fasting glucose & $36.6 \%$ \\
\hline Diabetes & $4.8 \%$ \\
\hline Abdominal obesity & $32.9 \%$ \\
\hline Elevated C-reactive protein & $19.2 \%$ \\
\hline Albumin-to-creatinine ratio, $\mathrm{mgg}^{-1}$ & $4.4(2.3-8.5)$ \\
\hline \multicolumn{2}{|l|}{ Clinic SBP, $\mathrm{mm} \mathrm{Hg}$} \\
\hline Mean & $115.2(10.9)$ \\
\hline Standard deviation (s.d.vvv) & $6.3(2.5)$ \\
\hline Average real variability $\left(\mathrm{ARV}_{\mathrm{vvv}}\right)$ & $7.2(3.2)$ \\
\hline \multicolumn{2}{|l|}{ Clinic $D B P, \mathrm{~mm} \mathrm{Hg}$} \\
\hline Mean & $74.7(7.3)$ \\
\hline Standard deviation (s.d.vvv) & $4.6(1.7)$ \\
\hline Average real variability (ARV $\mathrm{vvv})$ & $5.2(2.3)$ \\
\hline \multicolumn{2}{|l|}{ Ambulatory $S B P, \mathrm{~mm} \mathrm{Hg}$} \\
\hline Mean-24-h & $117.6(9.5)$ \\
\hline Mean-awake & $122.5(9.8)$ \\
\hline Mean-sleep & $105.0(10.3)$ \\
\hline Standard deviation (s.d.dn) & $8.8(1.8)$ \\
\hline Average real variability $\left(\mathrm{ARV}_{24}\right)$ & $8.4(2.1)$ \\
\hline \multicolumn{2}{|l|}{ Ambulatory $D B P, \mathrm{~mm} \mathrm{Hg}$} \\
\hline Mean-24-hour & $72.3(7.0)$ \\
\hline Mean-awake & $76.7(7.5)$ \\
\hline Mean-sleep & $61.0(7.6)$ \\
\hline Standard deviation (s.d.dn) & $7.4(1.5)$ \\
\hline Average real variability $\left(A R V_{24}\right)$ & $7.1(1.5)$ \\
\hline
\end{tabular}

Abbreviations: DBP, diastolic blood pressure; s.d., standard deviation; SBP, systolic blood pressure.

Impaired fasting glucose defined as hemoglobin A1c of $5.7 \%$ to $<6.5 \%$; diabetes was defined as $\geqslant 6.5 \%$ or anti-diabetes medication use; abdominal obesity is defined as a waist circumference $>35$ inches in women or $>40$ inches in men; elevated C-reactive protein defined as levels $>3 \mathrm{mgl}^{-1}$

${ }^{a}$ Except albuminuria, which is geometric mean (95\% confidence interval).

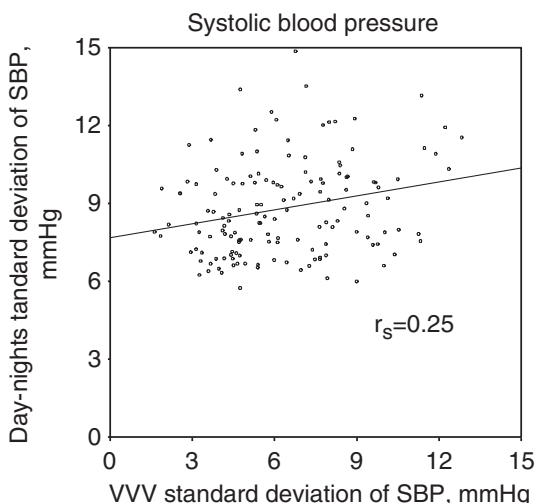

higher s.d..$_{\mathrm{vvv}}$ of SBP (Table 3). Although not statistically significant after further adjustment for diabetes status, abdominal obesity and elevated CRP, each doubling of albuminuria was associated with a value of $0.43 \mathrm{~mm} \mathrm{Hg}$ (standard error $=0.22, P=0.06$ ) higher s.d. ${ }_{\mathrm{vvv}}$ of SBP. Mean clinic SBP was associated with a higher

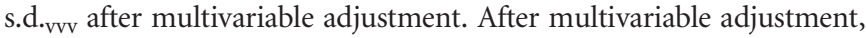
older age, being male, and higher mean 24-h SBP were each independently associated with higher s.d.dn of SBP. Only higher mean clinic DBP was associated with a higher s.d. .vvv $_{\text {of DBP. After }}$ adjustment for age, race, ethnicity, sex and mean 24-h DBP from ABPM, abdominal obesity was associated with a higher s.d.dn of DBP. After multivariable adjustment, older age was associated with a higher s.d.dn of DBP.

\section{Average real variability}

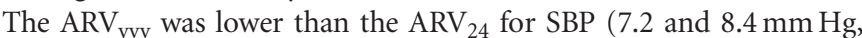
respectively; $P<0.001)$ and DBP $(5.2$ and $7.1 \mathrm{~mm} \mathrm{Hg}$, respectively; $P<0.001)$. The Spearman's correlation between $\mathrm{ARV}_{\mathrm{vvv}}$ and $\mathrm{ARV}_{24}$ was 0.17 for SBP and -0.13 for DBP (Supplementary Figure 1). The weighted Kappa statistics for quartiles of $\mathrm{ARV}_{\mathrm{vvv}}$ and $\mathrm{ARV}_{24}$ were 0.08 for SBP and -0.13 for DBP (Supplementary Table 1). For SBP, there was no association between being in the highest quartile of $A R V_{v v v}$ conditional on being in the highest quartile of $\mathrm{ARV}_{24}$ (observedto-expected ratio $=0.89,95 \%$ CI: 0.41-1.69). For DBP, individuals in the highest quartile of $\mathrm{ARV}_{24}$ were less likely to be in the highest quartile of $\mathrm{ARV}_{\mathrm{vvv}}$ (0.44, 95\% CI: 0.14-1.07). Hispanics had higher $\mathrm{ARV}_{\mathrm{vvv}}$ of SBP than whites, and elevated CRP was associated with higher $\mathrm{ARV}_{24}$ of SBP (Table 4). Mean clinic SBP was associated with higher $\mathrm{ARV}_{\mathrm{vvv}}$, and mean 24-h SBP was associated with higher $\mathrm{ARV}_{24}$. None of the factors investigated were associated with $\mathrm{ARV}_{\mathrm{vvv}}$ or $\mathrm{ARV}_{24}$ of DBP.

visit-to-visit and day-time standard deviation of blood pressure The Spearman's correlation of s.d.v .vv $_{\text {and }}$ s.d.day was 0.21 for SBP and 0.06 for DBP. The weighted Kappa statistic between s.d. .vvv $_{\text {and }}$ s.d.day was 0.05 for SBP, and between s.d. .vvv $_{\text {and }}$ s.d.day was 0.08 for DBP. The multivariable adjusted association of awake mean SBP with s.d.dn of SBP was similar to that observed for mean 24-h SBP described above (data not shown). After age, gender, race, ethnicity and full multivariable adjustment, awake mean DBP from ABPM was not associated with s.d.dn of DBP (data not shown).

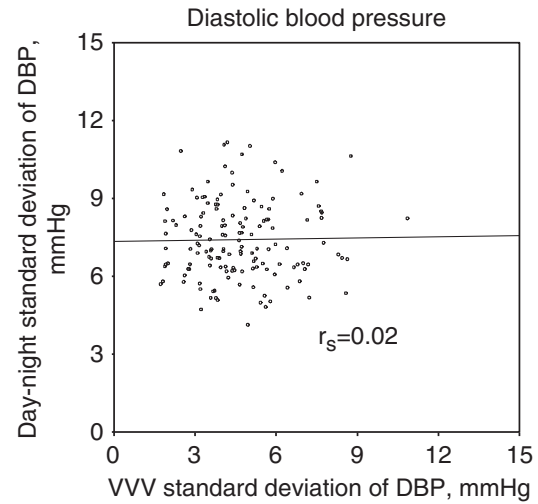

SBP - Systolic blood pressure

DBP - Diastolic blood pressure

Figure 1 Scatterplot of the visit-to-visit standard deviation (s.d.vvv) vs. day-night standard deviation (s.d.dn) from ambulatory blood pressure monitoring for systolic blood pressure (left panel) and diastolic blood pressure (right panel). 
Table 2 Cross tabulation of quartiles of visit-to-visit standard deviation (s.d.vvv) and day-night standard deviation (s.d.dn ${ }_{\text {) }}$ of systolic blood pressure (top panel) and diastolic blood pressure (bottom panel)

Quartile of day-night standard deviation of SBP from ambulatory monitoring (range in $\mathrm{mm} \mathrm{Hg}$ )

\begin{tabular}{|c|c|c|c|c|}
\hline & \multicolumn{4}{|c|}{ Quartile of day-night standard deviation of SBP from ambulatory monitoring (range in $\mathrm{mm} \mathrm{Hg}$ ) } \\
\hline & $1(<7.45)$ & $2(7.45-8.49)$ & $3(8.50-9.93)$ & $4(\geqslant 9.94)$ \\
\hline \multicolumn{5}{|c|}{ Quartile of VVV standard deviation of SBP (range in $\mathrm{mm} \mathrm{Hg}$ ) (range in $\mathrm{mm} \mathrm{Hg}$ ) } \\
\hline $1(<4.41)$ & 12 & 11 & 9 & 4 \\
\hline $2(4.41-5.95)$ & 10 & 10 & 10 & 7 \\
\hline $3(5.96-7.99)$ & 10 & 7 & 10 & 10 \\
\hline $4(\geqslant 8.00)$ & 4 & 9 & 8 & 15 \\
\hline
\end{tabular}

Quartile of day-night standard deviation of DBP from ambulatory monitoring (range in $\mathrm{mm} \mathrm{Hg}$ )

$1(<6.36) \quad 2(6.36-7.10) \quad 3(7.11-8.39) \quad 4(\geqslant 8.40)$

Quartile of VWV standard deviation of DBP (range in $\mathrm{mm} \mathrm{Hg}$ ) (range in $\mathrm{mm} \mathrm{Hg}$ )

$1(<3.40)$

$2(3.40-4.36)$

$3(4.37-5.62)$

9

$8-12$

$4(\geqslant 5.63)$

11

8

12
8

8
10

12

6

11

8

Numbers in table represent number of study participants.

Weighted Kappa statistics are 0.17 and 0.01 for SBP and DBP, respectively.

Observed-to-expected ratios for highest quartile are 1.66 (95\% Cl: 0.93-2.75) and 1.11 (95\% Cl: 0.56-1.98) for SBP and DBP, respectively.

Calculated via: http://www.sph.emory.edu/ cdckms/exact-midP-SMR.html.

VVV-visit-to-visit variability.

\section{visit-to-visit standard deviation and day-night change in blood} pressure

The Spearman's correlation of s.d.vvv and sleep-to-awake ratio in SBP and DBP was 0.07 and 0.05 , respectively. There were 19 (13\%), 98 $(67 \%)$ and $29(20 \%)$ participants with a sleep-to-awake ratio in SBP $<0.8$ (extreme dippers), 0.8 to $\leqslant 0.9$ (dippers) and $>0.9$ (non-

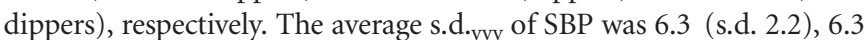
(s.d. 2.6) and $6.4 \mathrm{~mm} \mathrm{Hg}$ (s.d. 2.5) for participants with a sleep-toawake SBP ratio $<0.8, \quad 0.8$ to $\leqslant 0.9$, and $>0.9$, respectively $(P$ trend $=0.91)$. For DBP, $78(53 \%), 54(37 \%)$ and $14(10 \%)$ of participants had a sleep-to-awake ratio $<0.8,0.8$ to $\leqslant 0.9$ and $>0.9$, respectively. The average s.d. .vvv $_{\text {of DBP was }} 4.5$ (s.d. 1.5), 4.9 (s.d. 2.1) and $4.1 \mathrm{~mm} \mathrm{Hg}$ (SD 1.4) for participants with a sleep-to-awake DBP ratio $<0.8,0.8$ to $\leqslant 0.9$ and $>0.9$, respectively $(P$-trend $=0.77)$. After

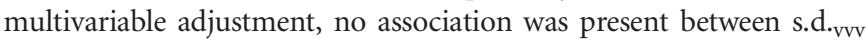
and sleep-to-awake ratio for SBP or DBP (data not shown).

\section{DISCUSSION}

In this study of people not taking antihypertensive medication, VVV of clinic BP and 24-h variability of BP on ABPM were only weakly correlated. The lack of a strong association between these two measures of BP variability was consistent for SBP and DBP, using s.d. and ARV as measures of variability. Furthermore, when VVV of BP and 24-h variability were grouped into quartiles, the degree of agreement between the levels of these markers was not significantly better than chance. ${ }^{18}$ Similarly, low correlations were present between visit-to-visit variability and awake $\mathrm{BP}$ variability and also day-night changes in BP. These data suggest that BP variability over $24 \mathrm{~h}$ from a single ABPM is not a proxy marker for VVV of BP obtained across multiple clinic visits, and that the mechanisms responsible for these indices of variability differ.

Studies have reported a strong association between VVV of SBP and stroke events. ${ }^{8}$ For example, comparing the highest to lowest deciles of the standard deviation (s.d) of SBP over seven visits in the UK-TIA trial, the hazard ratio for stroke was 6.22 (95\% CI: 4.16-9.29) after multivariable adjustment including mean SBP. The associations of VVV of SBP with both coronary heart disease (CHD) and all-cause mortality are also well established. ${ }^{8,9,20,21}$ Among 956 normotensive US adults with BP measured three times over 2 months in NHANES III, the multivariable adjusted hazard ratios for all-cause mortality associated with a s.d. of SBP of either $4.80-8.34 \mathrm{~mm} \mathrm{Hg}$ or $\geqslant 8.35 \mathrm{~mm} \mathrm{Hg}$, vs. $<4.80 \mathrm{~mm} \mathrm{Hg}$, were 1.57 (95\% CI: $1.07-2.18$ ) and 1.50 (95\% CI: $1.03-2.18$ ), respectively. ${ }^{9}$ In contrast, using data pooled from eleven studies on 8938 adults, Hansen et al. ${ }^{12}$ found a weak association (hazard ratios $<1.2$ ) between BP variability (for example, s.d.dn and $\mathrm{ARV}_{24}$ ) from ABPM and CVD outcomes. The discrepancy between VVV of BP, which maintains a strong association with outcomes, and shorter-term BP variability from ABPM is consistent with the findings of our study, which suggests that these two phenotypes may have a different underlying pathophysiology.

At least three recent studies have reported the correlation between VVV of BP and 24-h BP variability. ${ }^{8,22,23}$ In the BP lowering arm of the Anglo-Scandinavian Cardiac Outcomes Trial, the correlation between s.d. of daytime BP on ABPM and s.d.vvv for SBP was $0.26 .^{8}$ The authors of this study also reported that the coefficient of

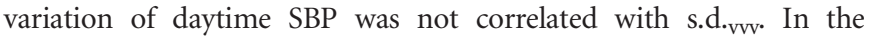
European Lacidipine Study on Atherosclerosis, Pearson's correlation coefficients between s.d. vvv $_{\text {of }}$ BP from $\geqslant$ seven visits and ABPM s.d. of $\mathrm{BP}$ were 0.20 and 0.15 for SBP and DBP, respectively. ${ }^{22}$ A similar correlation was reported between VVV of SBP, and sleep and awake ABP variability ( $r=0.19$ and 0.20 , respectively) in a study by Eguchi and colleagues. ${ }^{23}$ These modest correlations between VVV and 24-h variability are consistent with the current study. In contrast to prior studies, for the current study we conducted a more in-depth study of the relationship between VVV and 24-h variability. The association was weak between being in the highest quartiles of s.d.dn and s.d.vvv, as well as for the highest quartiles of $\mathrm{ARV}_{\mathrm{vvv}}$ and $\mathrm{ARV}_{24}$. These data suggest that $\mathrm{BP}$ variability on ABPM does not discriminate VVV of 
Table 3 Adjusted differences in visit-to-visit and day-night standard deviation of systolic blood pressure (top panel) and diastolic blood pressure (bottom panel) associated with participant characteristics

\begin{tabular}{|c|c|c|c|c|}
\hline & \multicolumn{2}{|c|}{ Age, gender, race, ethnicity and mean SBP adjusted } & \multicolumn{2}{|c|}{ Fully adjusted } \\
\hline & Visit-to-visit s.d. $\beta$ (s.e.) & Day-night s.d. $\beta$ (se.) & Visit-to-visit s.d. $\beta$ (s.e.) & Day-night s.d. $\beta$ (s.e.) \\
\hline \multicolumn{5}{|l|}{ Systolic blood pressure } \\
\hline Age, per 10 years & $0.28(0.23)$ & $0.59(0.15)^{* * *}$ & $0.23(0.24)$ & $0.50(0.16)^{* *}$ \\
\hline Men & $-0.45(0.46)$ & $0.64(0.30)^{*}$ & $-0.31(0.51)$ & $0.70(0.32)^{*}$ \\
\hline Black & $-0.62(0.83)$ & $-0.69(0.57)$ & $-0.62(0.89)$ & $-0.45(0.60)$ \\
\hline Hispanic & $1.44(0.83)$ & $-0.59(0.56)$ & $1.63(0.84)$ & $-0.53(0.58)$ \\
\hline \multicolumn{5}{|l|}{ Diabetes status } \\
\hline Normal fasting glucose & 0 (ref) & 0 (ref) & 0 (ref) & 0 (ref) \\
\hline Impaired fasting glucose & $0.37(0.44)$ & $0.45(0.30)$ & $0.27(0.45)$ & $0.36(0.30)$ \\
\hline Diabetes & $0.99(0.93)$ & $0.23(0.63)$ & $0.86(0.96)$ & $0.15(0.65)$ \\
\hline Abdominal obesity & $0.07(0.46)$ & $0.39(0.30)$ & $-0.01(0.52)$ & $0.44(0.34)$ \\
\hline Elevated c-reactive protein & $-0.12(0.52)$ & $-0.09(0.35)$ & $-0.32(0.59)$ & $-0.36(0.40)$ \\
\hline Albuminuria, per doubling of level & $0.47(0.21)^{*}$ & $0.22(0.15)$ & $0.43(0.22)$ & $0.17(0.15)$ \\
\hline Mean clinic SBP, per $10 \mathrm{~mm} \mathrm{Hg}$ & $0.85(0.22)^{* * *}$ & & $0.84(0.24)^{* * *}$ & \\
\hline Mean 24-hour SBP, per $10 \mathrm{~mm} \mathrm{Hg}$ & & $0.48(0.16)^{* *}$ & & $0.43(0.17)^{* *}$ \\
\hline \multicolumn{5}{|l|}{ Diastolic blood pressure } \\
\hline Age, per 10 years & $-0.16(0.15)$ & $0.35(0.13)^{* *}$ & $-0.16(0.16)$ & $0.29(0.14)^{*}$ \\
\hline Men & $-0.09(0.31)$ & $0.22(0.27)$ & $-0.11(0.34)$ & $0.36(0.28)$ \\
\hline Black & $0.66(0.58)$ & $0.22(0.51)$ & $0.64(0.63)$ & $0.12(0.54)$ \\
\hline Hispanic & $0.24(0.59)$ & $0.19(0.51)$ & $0.13(0.60)$ & $0.17(0.52)$ \\
\hline \multicolumn{5}{|l|}{ Diabetes status } \\
\hline Normal fasting glucose & 0 (ref) & 0 (ref) & 0 (ref) & 0 (ref) \\
\hline Impaired fasting glucose & $-0.07(0.31)$ & $0.25(0.27)$ & $-0.02(0.32)$ & $0.14(0.28)$ \\
\hline Diabetes & $0.66(0.67)$ & $0.89(0.58)$ & $0.83(0.69)$ & $0.68(0.59)$ \\
\hline Abdominal obesity & $0.10(0.33)$ & $0.67(0.26)^{*}$ & $0.24(0.37)$ & $0.58(0.30)$ \\
\hline Elevated c-reactive protein & $-0.14(0.37)$ & $0.46(0.31)$ & $-0.30(0.42)$ & $0.04(0.36)$ \\
\hline Albuminuria, per twofold higher level & $-0.17(0.15)$ & $0.15(0.13)$ & $-0.20(0.16)$ & $0.07(0.14)$ \\
\hline Mean clinic DBP, per $10 \mathrm{~mm} \mathrm{Hg}$ & $0.66(0.21)^{* *}$ & & $0.63(0.23)^{* *}$ & \\
\hline Mean 24-hour DBP, per $10 \mathrm{~mm} \mathrm{Hg}$ & & $0.12(0.18)$ & & $0.10(0.18)$ \\
\hline
\end{tabular}

Abbreviations: DBP, diastolic blood pressure; s.d., standard deviation; SBP, systolic blood pressure. $\beta$ (s.e.) is the difference in the standard deviation (standard error) from linear regression models. ${ }^{*} P<0.05,{ }^{* *} P<0.01,{ }^{* * *} P<0.001$

Fully adjusted includes all variables listed.

BP. The current study extends the prior studies of VVV and variability on ABPM to a multi-ethnic cohort of individuals without hypertension, and the consistency of the findings is noteworthy.

The low correlation between VVV of BP and 24-h BP variability is not entirely surprising given the hypothesized differences in their underlying mechanisms. ${ }^{23,24}$ There is a substantial amount of data showing physical and emotional stimulation result in BP variability over the course of a day. ${ }^{24}$ In contrast, it is recommended that clinic $\mathrm{BP}$ be measured in a controlled setting limiting the influence of external stimuli. ${ }^{14}$ At each of the six clinic visits used in the current study, BP was measured following the same study protocol, and quality control was monitored. There have been additional biologic underpinnings (for example, impaired sympathetic function, arterial stiffness and inflammation) hypothesized to affect both VVV of BP and 24-h BP variability. ${ }^{10,25,26}$ However, there is a paucity of data on these mechanisms, and on whether they have a similar effect on VVV of BP and 24-h BP variability.

Whereas mean SBP was associated with both s.d. ${ }_{\mathrm{vvv}}$ and s.d.dn, a few factors were not associated with both outcomes. The higher 24-h BP variability, but not VVV of BP, among older participants and men may be an indication that these participants experience greater variability in, or a heightened response to, physical activity, mental stress, or physical, behavioral or emotional factors over the ambulatory monitoring period. Although the differential effects of C-reactive protein and albuminuria on VVV of BP and 24-h BP variability are interesting, we urge caution in making definitive conclusions given the modest sample size of the current study. Future studies are needed to evaluate the different mechanisms that may underlie higher levels of VVV of BP and 24-h BP variability.

The results from the current study should be interpreted in the context of known and potential limitations. Although 886 participants completed the Masked Hypertension Study, only 20\% of participants were invited to complete the validation substudy. The duration of time between visits with BP measurements varied substantially, ranging from a median of 7 days between visits 1 and 2 to 165 days between visits 3 and 6 . Although a longer duration of time between visits 3 and 6 was planned, this may have affected the correlation between VVV of BP and 24-h variability of BP. Despite these limitations, the current study has several strengths. These include high quality control for the measurement of BP; the availability of three BP measurements per clinic visit; and the 
Table 4 Adjusted differences in visit-to-visit and 24-h average real variability of systolic blood pressure (top panel) and diastolic blood pressure (bottom panel) associated with participant characteristics

\begin{tabular}{|c|c|c|c|c|}
\hline & \multicolumn{2}{|c|}{ Age, gender, race, ethnicity and mean SBP adjusted } & \multicolumn{2}{|c|}{ Fully adjusted } \\
\hline & $A R V_{v v v} \beta$ (s.e.) & $A R V_{24} \beta$ (s.e.) & $A R V_{V V V} \beta$ (s.e.) & $A R V_{24} \beta$ (s.e.) \\
\hline Age, per 10 years & $0.34(0.29)$ & $0.22(0.19)$ & $0.30(0.31)$ & $0.19(0.20)$ \\
\hline Men & $-0.58(0.59)$ & $0.46(0.37)$ & $-0.57(0.65)$ & $0.59(0.40)$ \\
\hline Black & $-0.74(1.08)$ & $-0.74(0.71)$ & $-1.02(1.15)$ & $-0.46(0.74)$ \\
\hline Normal fasting glucose & 0 (ref) & 0 (ref) & 0 (ref) & 0 (ref) \\
\hline Impaired fasting glucose & $0.40(0.56)$ & $0.53(0.37)$ & $0.38(0.58)$ & $0.39(0.37)$ \\
\hline Diabetes & $1.47(1.20)$ & $0.35(0.79)$ & $1.38(1.24)$ & $-0.13(0.81)$ \\
\hline Abdominal obesity & $-0.36(0.60)$ & $0.30(0.38)$ & $-0.64(0.68)$ & $-0.16(0.42)$ \\
\hline Elevated c-reactive protein & $0.03(0.66)$ & $0.89(0.43)^{*}$ & $0.04(0.76)$ & $0.98(0.49)^{*}$ \\
\hline Albuminuria, per two fold higher level & $0.42(0.28)$ & $0.31(0.18)$ & $0.38(0.29)$ & $0.29(0.19)$ \\
\hline Age, per 10 years & $-0.22(0.21)$ & $0.09(0.14)$ & $-0.23(0.22)$ & $0.09(0.15)$ \\
\hline Men & $0.22(0.43)$ & $-0.05(0.29)$ & $0.25(0.47)$ & $0.11(0.30)$ \\
\hline Black & $0.86(0.80)$ & $0.08(0.54)$ & $0.84(0.86)$ & $0.09(0.58)$ \\
\hline Hispanic & $0.30(0.80)$ & $0.31(0.54)$ & $0.29(0.83)$ & $0.32(0.56)$ \\
\hline \multicolumn{5}{|l|}{ Diabetes status } \\
\hline Normal fasting glucose & $O$ (ref) & 0 (ref) & 0 (ref) & 0 (ref) \\
\hline Impaired fasting glucose & $-0.04(0.43)$ & $0.05(0.29)$ & $-0.02(0.44)$ & $-0.06(0.30)$ \\
\hline Diabetes & $0.98(0.91)$ & $0.66(0.62)$ & $1.16(0.94)$ & $0.35(0.63)$ \\
\hline Abdominal obesity & $0.09(0.45)$ & $0.40(0.28)$ & $0.29(0.51)$ & $0.19(0.32)$ \\
\hline Elevated c-reactive protein & $-0.35(0.50)$ & $0.58(0.33)$ & $-0.62(0.58)$ & $0.44(0.39)$ \\
\hline Albuminuria, per two fold higher level & $0.0(0.21)$ & $0.19(0.14)$ & $-0.03(0.22)$ & $0.16(0.15)$ \\
\hline
\end{tabular}

Abbreviations: DBP, diastolic blood pressure; s.d., standard deviation; SBP, systolic blood pressure. $\beta$ (s.e.) is the difference in the standard deviation (standard error) from linear regression models. ${ }^{*} P<0.05,{ }^{* *} P<0.01,{ }^{* * *} P<0.001$.

Fully adjusted includes all variables listed.

collection of a broad array of potential correlates of high BP variability.

The correlation between VVV of BP and 24-h BP variability from ABPM was low in the current study. As VVV of BP requires multiple visits and may not be practical, or in some cases feasible, data from the current study suggest that using BP variability from ABPM will not provide similar information. Future studies are needed to evaluate practical approaches for measuring VVV of BP across multiple clinic visits.

\section{CONFLICT OF INTEREST}

The authors declare no conflict of interest.

\section{ACKNOWLEDGEMENTS}

We are indebted to the study participants and research staff of the Masked Hypertension Study, without whose cooperation and dedication this study would not have been possible. This work was supported by grants P01HL47540 (PI: J Schwartz) and R24-HL076857 (PI: K Davidson) from the National Heart, Lung, and Blood Institute. The research was also supported in part by General Clinical Research Center grant MO1-RR10710 (Stony Brook University) and CTSA grant UL1 TR000040 (formerly UL1 RR024156; Columbia University) from NCATS-NCRR/NIH.

\section{DISCLAIMER}

The content is solely the responsibility of the authors and does not necessarily represent the official view of the $\mathrm{NIH}$

1 Staessen JA, Beilin L, Parati G, Waeber B, White W. Task force iv: Clinical use of ambulatory blood pressure monitoring. Participants of the 1999 consensus conference on ambulatory blood pressure monitoring. Blood pressure monitoring 1999; 4: 319-331.

2 Pickering TG, Shimbo D, Haas D. Ambulatory blood-pressure monitoring. N Engl J Med 2006; 354: 2368-2374.

3 Salles GF, Cardoso CR, Muxfeldt ES. Prognostic influence of office and ambulatory blood pressures in resistant hypertension. Arch Intern Med 2008; 168 : 2340-2346.

4 Clement DL, De Buyzere ML, De Bacquer DA, de Leeuw PW, Duprez DA, Fagard RH, Gheeraert PJ, Missault LH, Braun JJ, Six RO, Van Der Niepen P, O'Brien EOffice versus Ambulatory Pressure Study I. Prognostic value of ambulatory blood-pressure recordings in patients with treated hypertension. N Engl J Med 2003; 348: 2407-2415.

5 Staessen JA, Thijs L, Fagard R, O'Brien ET, Clement D, de Leeuw PW, Mancia G, Nachev C, Palatini P, Parati G, Tuomilehto J, Webster J. Predicting cardiovascular risk using conventional vs ambulatory blood pressure in older patients with systolic hypertension. Systolic hypertension in europe trial investigators. JAMA 1999; 282 : 539-546.

6 National institute for health and clinical excellence. Nice clinical guideline 127. Hypertension: Clinical management of primary hypertension in adults 2011.

7 Lovibond K, Jowett S, Barton P, Caulfield M, Heneghan C, Hobbs FD, Hodgkinson J, Mant J, Martin U, Williams B, Wonderling D, McManus RJ. Cost-effectiveness of 
options for the diagnosis of high blood pressure in primary care: A modelling study. Lancet 2011; 378: 1219-1230.

8 Rothwell PM, Howard SC, Dolan E, O'Brien E, Dobson JE, Dahlof B, Sever PS, Poulter NR. Prognostic significance of visit-to-visit variability, maximum systolic blood pressure, and episodic hypertension. Lancet 2010; 375: 895-905.

9 Muntner P, Shimbo D, Tonelli M, Reynolds K, Arnett DK, Oparil S. The relationship between visit-to-visit variability in systolic blood pressure and all-cause mortality in the general population: Findings from nhanes iii, 1988 to 1994. Hypertension 2011; 57 160-166.

10 Rothwell PM. Limitations of the usual blood-pressure hypothesis and importance of variability, instability, and episodic hypertension. Lancet 2010; 375: 938-948.

11 Oparil S. New challenges in blood pressure goals and assessment. Nat Clin Pract Cardiovasc Med 2011; 8: 1-2.

12 Hansen TW, Thijs L, Li Y, Boggia J, Kikuya M, Bjorklund-Bodegard K, Richart T, Ohkubo T, Jeppesen J, Torp-Pedersen C, Dolan E, Kuznetsova T, Stolarz-Skrzypek K, Tikhonoff V, Malyutina S, Casiglia E, Nikitin Y, Lind L, Sandoya E, Kawecka-Jaszcz K, Imai Y, Wang J, Ibsen H, O'Brien E, Staessen JA. Prognostic value of reading-to-reading blood pressure variability over $24 \mathrm{~h}$ in 8938 subjects from 11 populations. Hypertension 2010; 55: 1049-1057.

13 International Expert C. International expert committee report on the role of the alc assay in the diagnosis of diabetes. Diabetes Care 2009; 32: 1327-1334.

14 Pickering TG, Hall JE, Appel LJ, Falkner BE, Graves J, Hill MN, Jones DW, Kurtz T, Sheps SG, Roccella EJ. Recommendations for blood pressure measurement in humans and experimental animals: Part 1: Blood pressure measurement in humans: a statement for professionals from the subcommittee of professional and public education of the american heart association council on high blood pressure research. Hypertension 2005; 45: 142-161.

15 Muntner P, Joyce C, Levitan EB, Holt E, Shimbo D, Webber LS, Oparil S, Re R, Krousel-Wood M. Reproducibility of visit-to-visit variability of blood pressure measured as part of routine clinical care. J Hypertens 2011; 29: 2332-2338.

16 Bilo G, Giglio A, Styczkiewicz K, Caldara G, Maronati A, Kawecka-Jaszcz K, Mancia G, Parati G. A new method for assessing 24-h blood pressure variability after excluding the contribution of nocturnal blood pressure fall. J Hypertens 2007; 25: 2058-2066.
17 Mena L, Pintos S, Queipo NV, Aizpurua JA, Maestre G, Sulbaran T. A reliable index for the prognostic significance of blood pressure variability. J Hypertens 2005; 23: 505-511.

18 Landis JR, Koch GG. An application of hierarchical kappa-type statistics in the assessment of majority agreement among multiple observers. Biometrics 1977; 33: 363-374.

19 Kario K, Matsuo T, Kobayashi H, Imiya M, Matsuo M, Shimada K. Nocturnal fall of blood pressure and silent cerebrovascular damage in elderly hypertensive patients. Advanced silent cerebrovascular damage in extreme dippers. Hypertension 1996; 27: 130-135.

20 Grove JS, Reed DM, Yano K, Hwang LJ. Variability in systolic blood pressure-a risk factor for coronary heart disease? Am J Epidemiol 1997; 145: 771-776.

21 Hsieh YT, Tu ST, Cho TJ, Chang SJ, Chen JF, Hsieh MC. visit-to-visit variability in blood pressure strongly predicts all-cause mortality in patients with type 2 diabetes: A 5.5year prospective analysis. Eur J Clin Invest 2012; 42: 245-253.

22 Mancia G, Facchetti R, Parati G, Zanchetti A. visit-to-visit blood pressure variability in the European Lacidipine Study on Atherosclerosis: methodological aspects and effects of antihypertensive treatment. J Hypertens 2012; 30: 1241-1251.

23 Eguchi K, Hoshide S, Schwartz JE, Shimada K, Kario K. visit-to-visit and ambulatory blood pressure variability as predictors of incident cardiovascular events in patients with hypertension. Am J Hypertens 2012; 25: 962-968.

24 Mancia G. Short- and long-term blood pressure variability: Present and future. Hypertension 2012; 60: 512-517.

25 Tatasciore A, Zimarino M, Renda G, Zurro M, Soccio M, Prontera C, Emdin M, Flacco M, Schillaci G, De CR. Awake blood pressure variability, inflammatory markers and target organ damage in newly diagnosed hypertension. Hypertens Res 2008; 31 2137-2146.

26 Diaz KM, Veerabhadrappa P, Kashem MA, Feairheller DL, Sturgeon KM, Williamson ST Crabbe DL, Brown MD. Relationship of visit-to-visit and ambulatory blood pressure variability to vascular function in african americans. Hypertens Res 2012; 35: 55-61.

Supplementary Information accompanies the paper on Hypertension Research website (http://www.nature.com/hr) 